Document downloaded from:

http://hdl.handle.net/10251/120702

This paper must be cited as:

Barrera Vilar, D.; Madrigal-Madrigal, J.; Sales Maicas, S. (2018). Long Period Gratings in Multicore Optical Fibers for Directional Curvature Sensor Implementation. Journal of Lightwave Technology. 36(4):1063-1068. https://doi.org/10.1109/JLT.2017.2764951

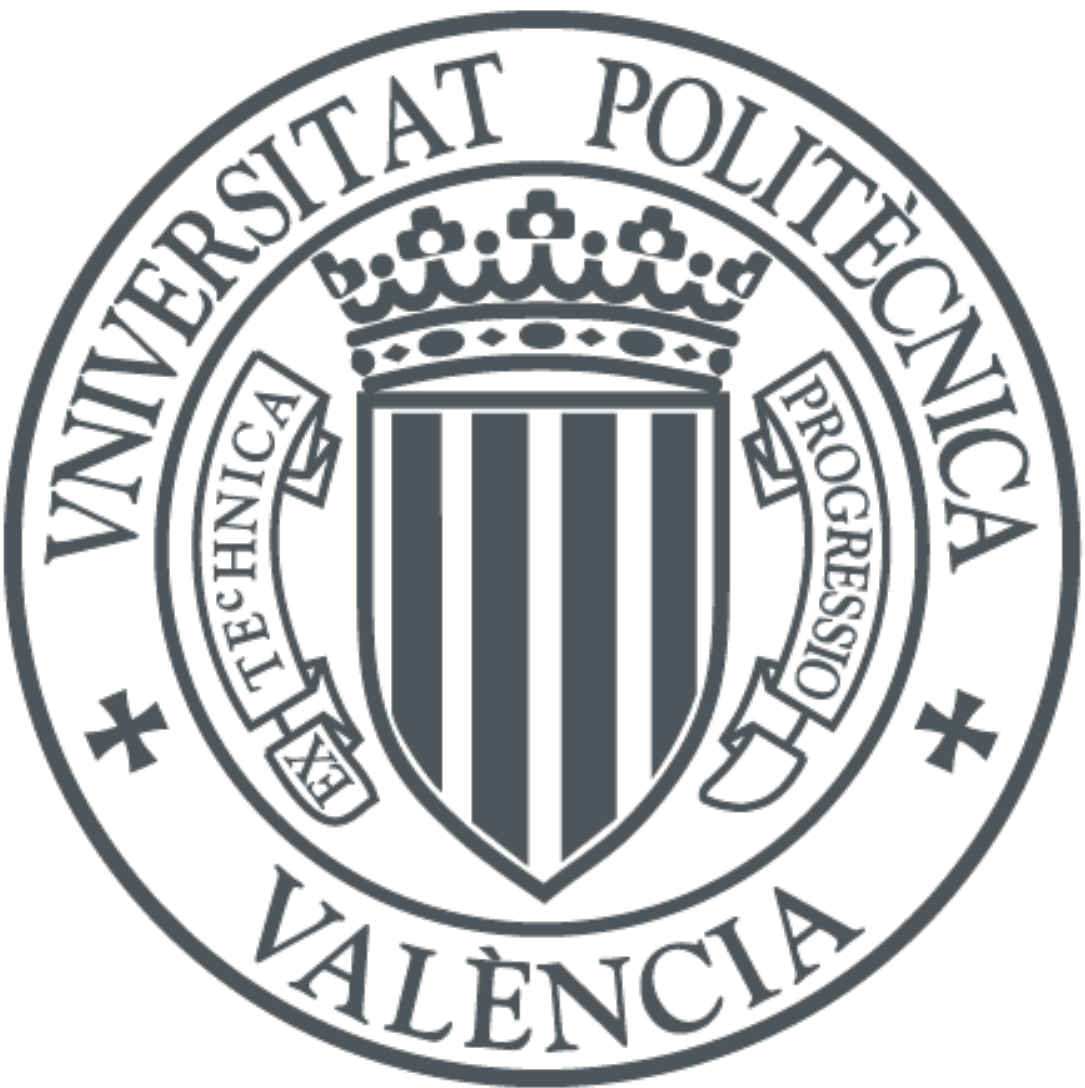

The final publication is available at

http.//doi.org/10.1109/JLT.2017.2764951

Copyright Institute of Electrical and Electronics Engineers

Additional Information 


\title{
Long period gratings in multicore optical fibers for directional curvature sensor implementation
}

\author{
David Barrera, Javier Madrigal, and Salvador Sales, Senior Member, IEEE
}

\begin{abstract}
Multicore optical fibers are especially attractive for the fabrication of curvature and shape sensors due to the spatial distribution of the different cores. Fiber Bragg gratings have been used in the past for the implementation of these sensors, however, despite their inherent properties, they have a very limited sensitivity. In this paper, we study the use of long period gratings (LPGs) for the implementation of a directional curvature sensor. We inscribed a set of three different LPGs in a seven core optical fiber using a selective inscription technique. We inscribed a single LPG in the external cores and an array of three LPGs in the central core. We have characterized the proposed sensor for strain, torsion, and curvature magnitude and direction. The proposed sensor shows a linear response for curvature magnitudes from $0 \mathrm{~m}^{-1}$ to $1.77 \mathrm{~m}^{-1}$ with a maximum curvature sensitivity of $-\mathbf{- 4 . 8 5} \mathrm{nm} / \mathrm{m}^{-1}$ and shows a near sinusoidal behavior in all the cores with curvature directions from $0^{\circ}$ to $360^{\circ}$. The sensor shows a good insensitivity to strain. The torsion in the MCF can be detected and measured using the maximum attenuation of the LPGs in the external cores.
\end{abstract}

Index Terms - Long Period Gratings, Multicore optical fiber, Optical fiber sensor, curvature.

\section{INTRODUCTION}

$\mathrm{F}$ iber optic sensors offer a series of advantages over traditional electrical sensors, such as non-conductive, resistant to hard environmental conditions or immunity to electromagnetic interference. Additionally, the small size, the lightweight and the flexibility permit to embed the optical fiber sensors into a structure in order to monitor important structural health parameters like strain, temperature or curvature. These properties make optical fiber sensors a key component in many industrial processes, civil engineering applications or aerospace industry. The measurement of curvature is of special importance in structural health monitoring for civil engineering and aerospace industry. Fiber Bragg gratings, long period gratings (LPGs) and interferometers have been proposed for the implementation of

This work was supported by the Sistema Nacional de Garantía Juvenil grant PEJ-2014-A-75865 (Promoción de Empleo Joven e Implantación de la Garantía Juvenil 2014, MINECO), the Ministry of Economy and Competitiveness under the project TEC2014-60378-C2-1-R, the support of FINESSE the European Union's Horizon 2020 research and innovation programme under the Marie Sklodowska-Curie Action grant agreement $\mathrm{n}^{\circ} 722509$, and the support of the Generalitat Valenciana under the grant APOSTD/2016/015.

D. Barrera, J. Madrigal and S. Sales are with the ITEAM research institute, Universitat Politècnica de València, Camino de Vera s/n, 46022, Valencia, Spain (e-mail: dabarvi@iteam.upv.es). curvature sensors $[1,2]$. In general, the use of a single core optical fiber limits the measurement of the bending direction due to the symmetry of the fiber. In order to achieve a directional curvature sensor several methods have been proposed, for example special shape fibers, cladding index modification and multicore optical fibers (MCF) [3-5]. In particular, MCFs have attracted considerable interest in the last years due to their potential in many applications including broadband communications, microwave photonics, and optical sensing [7, 11]. In optical sensing, MCFs are especially attractive for curvature and shape sensing because the spatial distribution of the different cores produces a differential strain in the cores when the fiber is curved. The use of FBGs has been proposed for measure the curvature magnitude and the curvature direction along the optical fiber [12-16]. However, despite the advantages of FBGs in optical sensing, their sensitivity is very limited. In this paper, we explore the use of long period gratings (LPG) inscribed in MCF for the implementation of a complete directional curvature sensor [17]. In contrast to previous works, the use of LPGs can offer a higher sensitivity compared with the use of FBGs. Moreover, the selective inscription in the cores of a MCF allows the fabrication of an array of three different LPGs along the optical fiber with only one LPG inscribed in each of the outer cores reducing the number of attenuation bands and decreasing the complexity of the optical spectrum.

\section{GRATING INSCRIPTION}

The sensor has been implemented on a seven core optical fiber from Fibercore Ltd. The MCF has a cladding diameter of $125 \mu \mathrm{m}$ and the cores are spatially distributed in a hexagonal pattern with one of the cores in the center. The core spacing or pitch is $35 \mu \mathrm{m}$ and each core has a mode field diameter of $6.4 \mu \mathrm{m}$ and a numerical aperture of 0.2. The MCF was hydrogen-loaded at ambient temperature for two weeks at 50 bar pressure to increase its photosensitivity. The inscription of the LPGs was made point by point using a $244 \mathrm{~nm} \mathrm{CW}$ frequency doubled argon-ion laser with an output power of $60 \mathrm{~mW}$. Two fain-in/out devices were spliced at both ends of the MCF in order to access the cores individually during the inscription and the later characterization.

Fig. 1 shows a schematic view of the inscription setup which uses two rotation stages to align the cores in the MCF and a closed loop core tracking system. The core tracking subsystem is based on the photoluminescence generated by the 


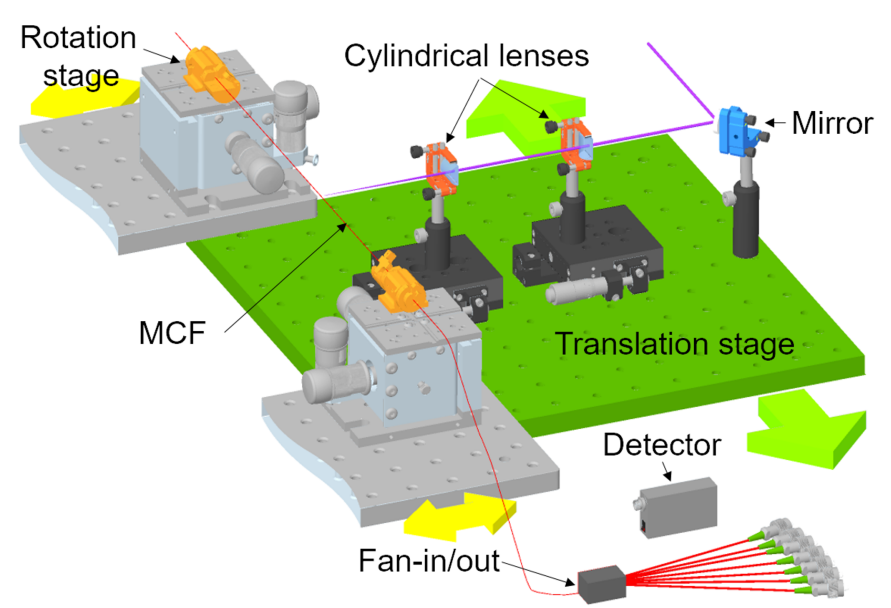

Fig. 1. Inscription setup used for the inscription of the LPGs.

UV radiation $[18,19]$ and maintains the laser beam directed towards the core of interest providing information about the spatial position of the different cores within the cross-sectional area of the optical fiber. The setup can be used to inscribe simultaneously in all the cores, in a group of cores or just in a specific core controlling the laser beam dimensions and having a precise knowledge of the spatial position of each core inside the MCF. For the inscription of the devices shown in this article the laser beam dimensions were adapted to inscribe the LPGs in a single row of cores.

Before the inscription, the central core and two external cores were aligned in the center of the fiber using the rotation stages and the tracking system. The alignment is repeated at several locations along the MCF correcting any twist of the optical fiber. Successive 60 degree rotations of the MCF allows inscribing in the other planes of the optical fiber, see Fig. 2(a).

Fig. 2(b) shows the scheme of the implemented curvature sensor. It is composed by three LPGs inscribed in the three planes of the MCF. The periods of the inscribed LPGs are $300 \mu \mathrm{m}, 300.95 \mu \mathrm{m}$, and $298.63 \mu \mathrm{m}$. The whole inscription process results in a central core with three $30 \mathrm{~mm}$ long LPGs whereas the spectra of the outer cores have only one inscribed LPG. In the central core, there are three main attenuation bands located at $1555.7 \mathrm{~nm}, 1557.08 \mathrm{~nm}$ and $1559.9 \mathrm{~nm}$. The outer cores show a main attenuation band at wavelengths between $1552 \mathrm{~nm}$ and $1576 \mathrm{~nm}$. Even with the same period the LPG Bragg wavelength of the opposite cores do not match due to differences in the UV irradiation during the inscription.

In order to illustrate the differences in the optical spectrum between the central core and the external cores Fig. 3 shows the spectra of the inscribed LPG in the central core (core \#1) and the two aligned external cores (core \#4 and core \#7) after the first inscription process $(300 \mu \mathrm{m}$ period). Extending the wavelength span, central core exhibits two main attenuation bands located at $1483 \mathrm{~nm}$ and $1557.08 \mathrm{~nm}$ whereas the spectra of the two external cores show a main attenuation band at $1560.1 \mathrm{~nm}$ and at $1569.4 \mathrm{~nm}$, respectively, and multiple weaker attenuation bands along the optical spectrum. The differences between the central core and the external cores spectra are explained due to the spatial distribution of the

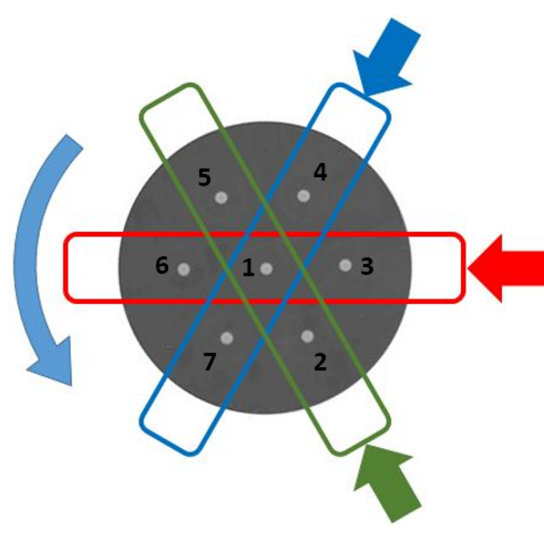

(a)
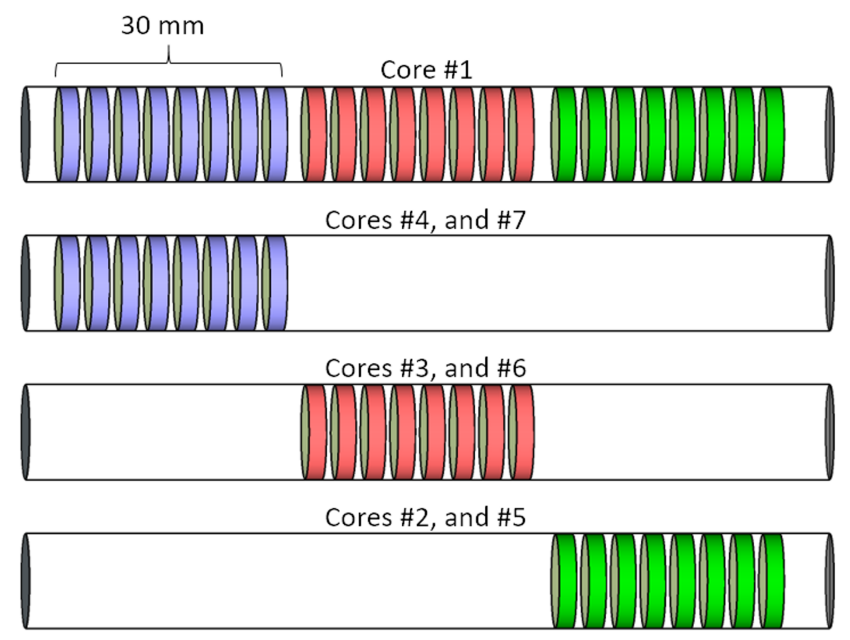

(b)

Fig. 2. (a) Cross-section of MCF and defined core planes for the implementation of the curvature sensor. (b) Scheme of the LPGs inscribed in each of the cores of the MCF.

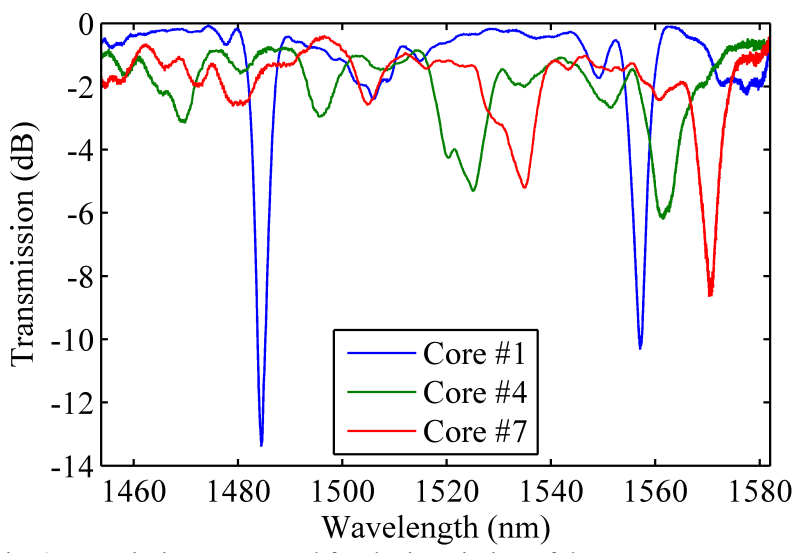

Fig. 3. Inscription setup used for the inscription of the LPGs.

cores inside the optical fiber. The central wavelength of the LPGs depends on the period of the LPG $(\Lambda)$ and the difference between the effective refractive index of the core ( $\left.n_{\text {core }}\right)$ and the effective refractive index of one of the cladding modes $\left(n_{\text {clad }}^{(i)}\right)[20,21]$. 


$$
\lambda_{i}=\Lambda\left[n_{\text {core }}-n_{\text {clad }}^{(i)}\right]
$$

In MCFs whereas the cladding modes for the central core are similar to those in single core fibers, the cladding modes distribution of the external cores is altered by the asymmetry of the optical fiber. This particular property gives the MCFs an extraordinary potential in optical sensing since different cores can significantly differ in their sensitivities if the period of the grating is properly selected.

\section{EXPERIMENTAL RESULTS}

We characterized the curvature sensor analyzing the behavior of the main attenuation bands of the LPGs located around $1560 \mathrm{~nm}$ for strain, torsion, curvature magnitude and curvature direction. Fig. 4 shows the setup used for the complete sensor characterization.

\section{A. Strain}

The curvature sensor was first characterized under strain using the setup shown in Fig. 4(a). The sides of the MCF were clamped to the two translation stages with the two fiber rotators in a fixed position and the strain was progressively increased to a maximum of $1400 \mu \varepsilon$ in steps of $140 \mu \varepsilon$. Fig. 5 shows the wavelength shift of the central core and two opposite external cores. In all the cores the wavelength shift

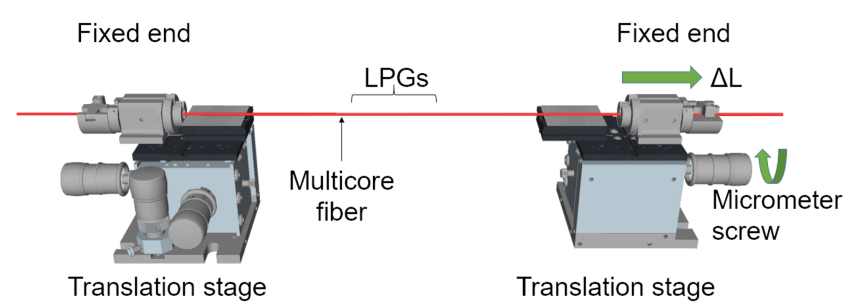

(a)

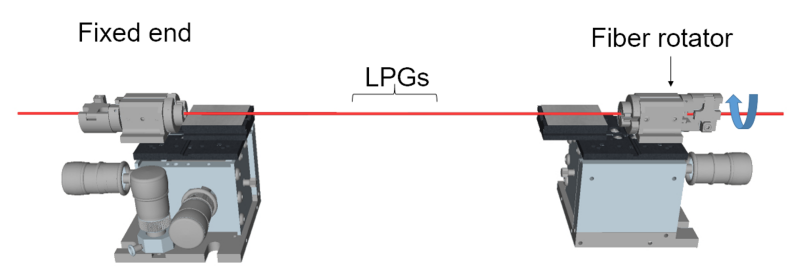

(b)

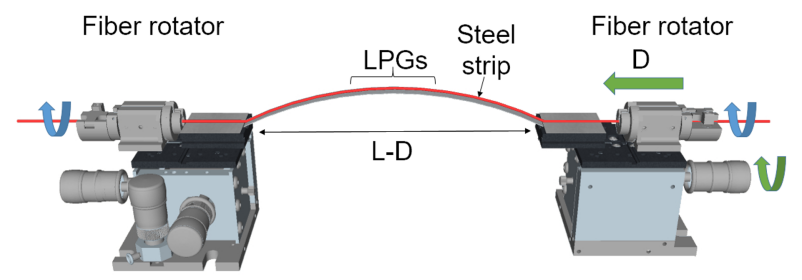

(c)

Fig. 4. Characterization setup for the curvature sensor. (a) Strain characterization. (b) Torsion characterization. (c) Curvature magnitude and direction characterization.

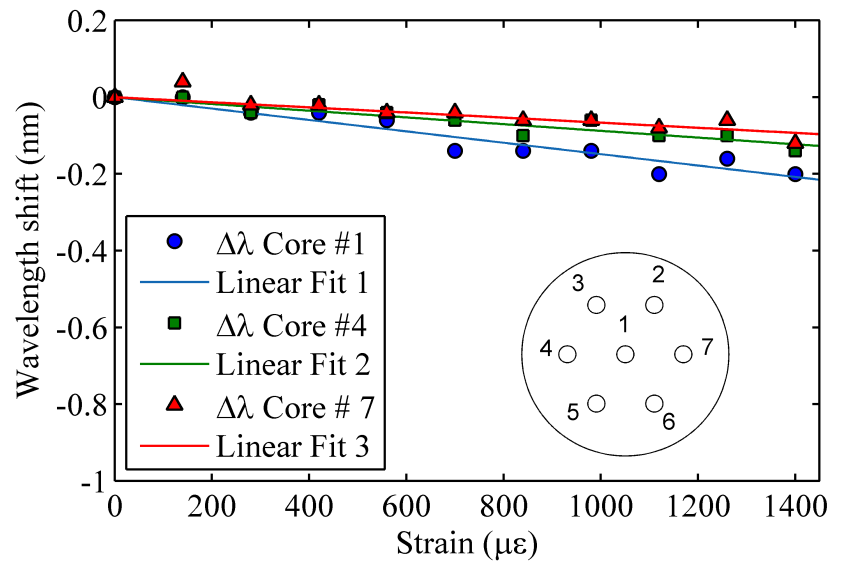

Fig. 5. Wavelength shift of the central and two external cores of the MCF under strain.

follows a linear behavior with the applied strain being the wavelength shift lower than $0.21 \mathrm{~nm}$ for the maximum strain applied.

\section{B. Torsion}

The position of the cores inside the MCF is very important for a curvature sensor since the curvature direction is determined as an angle relative to the position of the cores. In shape sensors a twist in the optical fiber can lead to errors in the curvature direction determination. The effect of a fiber twist in the proposed curvature sensor was characterized using the setup depicted in Fig. 4(b). The translation stage was placed in the initial position and one of the fiber rotators was released for changing the angle of one of the MCF ends. The fiber rotator angle was increased from $0^{\circ}$ to $360^{\circ}$ in 90 degree steps producing a maximum twist rate of $26.18 \mathrm{rad} / \mathrm{m}$. Fig. 6(a) depicts the evolution of the LPG spectrum of two opposite cores of the MCF, core \#3 and core \#6 showing a change in the level of the LPG attenuation. Fig. 6(b) compares the maximum attenuation at the Bragg wavelength for cores $\# 1, \# 3$, and \#6 with the torsion. The twist of the fiber produces an opposite behavior for central core and external cores. The LPGs in the external cores shows a reduction of their level when the twist angle increases whereas the LPG level in the central core is slightly increased. Torsion also produces a small wavelength shift that is similar in magnitude to that shown in the strain characterization test with a maximum shift of $0.2 \mathrm{~nm}$ making possible to determine the torsion of the fiber just measuring the attenuation at the Bragg wavelength. The experimental results have been fitted to second order polynomials in order to determine the torsion of the fiber. Equations (2) to (4) show the fitting polynomials with $\theta$ being the twist angle and $P_{1}, P_{3}$ and $P_{6}$ the measured attenuation at the Bragg wavelength:

$$
\begin{gathered}
P_{1}(\theta)=-1.09 \cdot 10^{-5} \cdot \theta^{2}-6.35 \cdot 10^{-5} \cdot \theta-11.56 \\
P_{3}(\theta)=5.24 \cdot 10^{-5} \cdot \theta^{2}-2.7 \cdot 10^{-3} \cdot \theta-9.3 \\
P_{6}(\theta)=5 \cdot 10^{-5} \cdot \theta^{2}+6.78 \cdot 10^{-4} \cdot \theta-10.33
\end{gathered}
$$




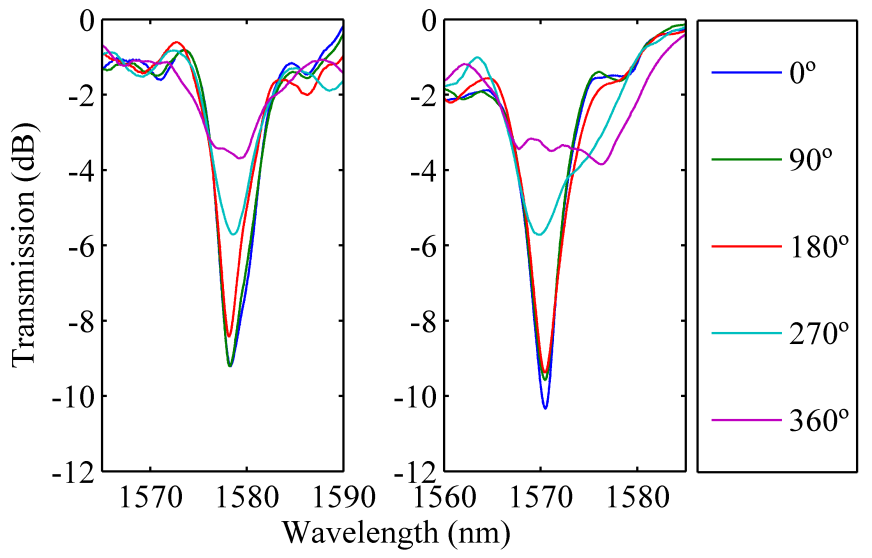

(a)

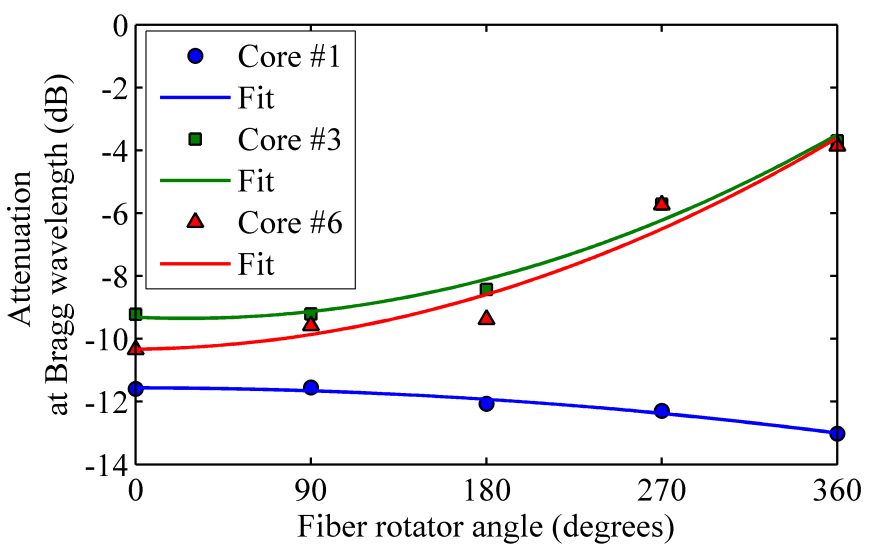

(b)

Fig. 6. (a) Evolution of the LPG spectrum for cores \#3 and core \#6. (b) Maximum attenuation at Bragg wavelength for cores \#1, core \#3, and core \#6 and respective second order polynomial fitting.

\section{Curvature}

The curvature response was characterized using the setup of the Fig. 4(c). A calibrated steel strip of $0.3 \mathrm{~mm}$ thickness was installed between the two translation stages. The fiber sensor was then placed at the top of the steel strip setting the LPGs at the center of the metal strip. The movement of the translation stage reduces the distance between both ends bending the steel strip. For small curvatures the shape of the steel strip can be approximated by the arc of a circle. The curvature of the steel strip $C$, can be calculated by equation (5) where $L$ is the length of the steel strip and $D$ corresponds to the distance traveled by the translation stage. In this setup, $L=197 \mathrm{~mm}$ and $D$ can be set from $0 \mathrm{~mm}$ to $13 \mathrm{~mm}$ by steps of $0.01 \mathrm{~mm}$.

$$
\sin \left(\frac{L C}{2}\right)=\frac{(L-D) C}{2}
$$

To study the sensor performance for determining the curvature magnitude and curvature direction two independent experimental tests were performed. For both experiments the transmission optical spectrum of all the cores were collected.

The experimental characterization of the curvature magnitude sensitivity was carried out by first fixing the

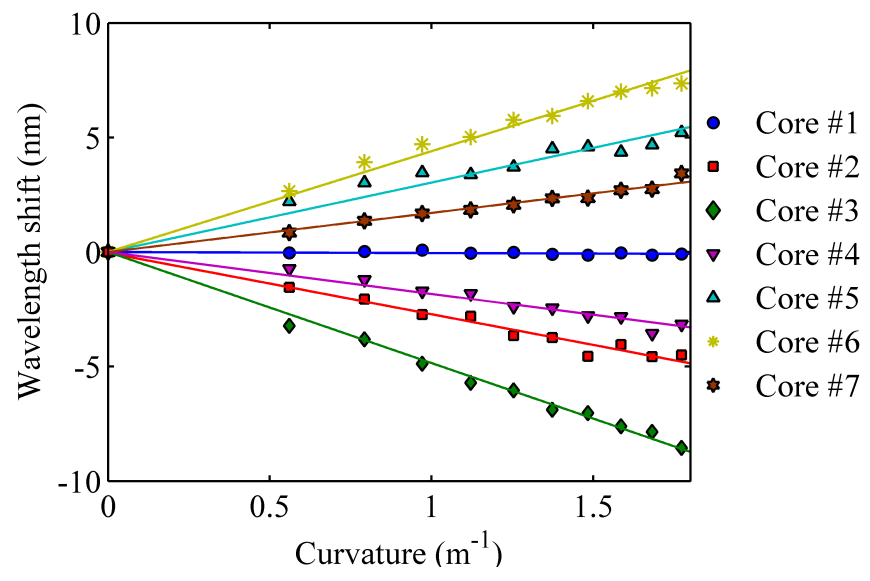

(a)

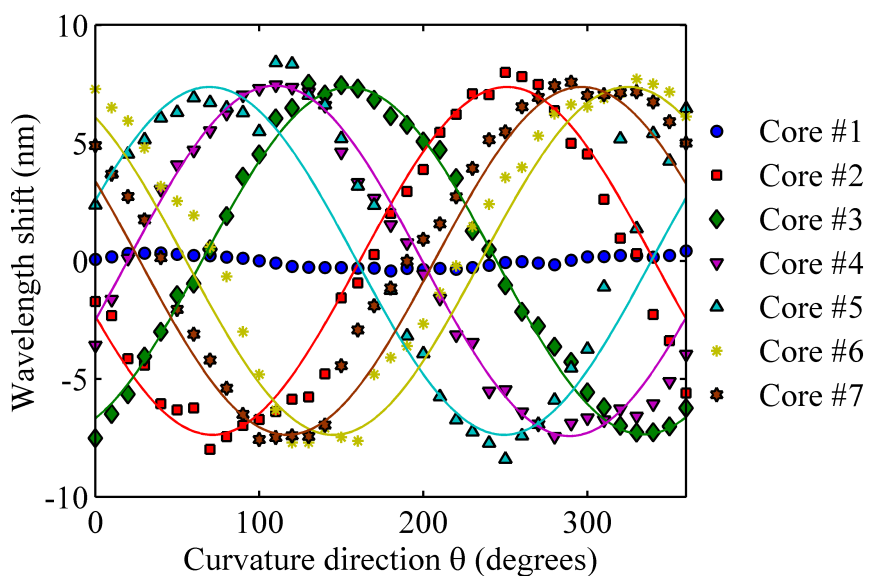

(b)

Fig. 8. (a) Wavelength shift with the curvature magnitude. (b) Wavelength shift with curvature direction at a constant curvature of $1.25 \mathrm{~m}^{-1}$

curvature direction with the fiber rotation stages and then moving the translation stage in steps of $0.1 \mathrm{~mm}$. The maximum induced curvature magnitude was $1.77 \mathrm{~m}^{-1}$. Fig. 8(a) shows the wavelength shift of each LPG relative to the wavelength at the straight position. The results show a linear relationship between the wavelength shift and the curvature applied. Each core shows a different curvature sensitivity that is consequence of their relative position inside the MCF. Central core shows a negligible wavelength shift whereas the outer cores show different curvature sensitivities: Core \#2 has a sensitivity of $-2.68 \mathrm{~nm} / \mathrm{m}^{-1}$, core $\# 3$ has a sensitivity of $-4.85 \mathrm{~nm} / \mathrm{m}^{-1}$, core $\# 4$ has a sensitivity of $-1.83 \mathrm{~nm} / \mathrm{m}^{-1}$, core \#5 has a sensitivity of $3.03 \mathrm{~nm} / \mathrm{m}^{-1}$, core \#6 has a sensitivity of $4.40 \mathrm{~nm} / \mathrm{m}^{-1}$, and core $\# 7$ has a sensitivity of $1.70 \mathrm{~nm} / \mathrm{m}^{-1}$. The maximum sensitivity is reached when the cores are aligned with the curvature direction.

In the second experimental test we have studied the curvature direction. The steel strip was bended at a constant curvature of $1.25 \mathrm{~m}^{-1}$. The fiber was then rotated in 10 degree steps using the two rotation stages at the same time for curvature directions from $0^{\circ}$ to $360^{\circ}$. Because each core occupies a different initial position inside the MCF Fig. 8(b) shows the wavelength shift of the LPG when the fiber is 
rotated relative to the mean of the complete wavelength shift range of each LPG. The results in each core show a good agreement with a sinusoidal behavior. It can be observed that the experimental data differs in some cores from the fitting curves near the maximum wavelength shift which is caused by the effect of spectrum notch splitting [22].

The relationship between the wavelength shifts in each core is unique for any curvature direction and is constant since the sensor shows a linear response with the curvature magnitude. Once the curvature direction is determined, the curvature magnitude is proportional to the wavelength shift. This makes possible to determine at the same time curvature magnitude and direction as long as the fiber is not twisted which can be guaranteed with a proper packaging. Under a known or predictable curvature direction the sensor can be employed as a distributed sensor, being able to measure non-uniform curvatures.

\section{CONCLUSION}

We have successfully inscribed selectively LPGs in a seven core MCF obtaining different LPGs in each of the outer cores along the optical fiber. The geometry of the MCF produces different LPGs in the central core and the outer cores. The proposed sensor has been characterized under strain, torsion and curvature magnitude and direction. The sensor shows a good insensitivity to strain. We have shown that torsion produces a reduction of the LPG attenuation band only in the external cores and this change in the LPG can be used to determine the twist of the MCF. Finally, we have demonstrated the ability of the sensor to measure different curvature magnitudes from $0 \mathrm{~m}^{-1}$ to $1.77 \mathrm{~m}^{-1}$ and curvature directions from $0^{\circ}$ to $360^{\circ}$. The outer cores show a linear wavelength shift with the curvature magnitude whereas the central core shows a negligible wavelength shift. For constant curvatures and changing the curvature direction the LPGs in the outer cores shows a near sinusoidal pattern demonstrating that the sensitivity of the cores to the curvature magnitude depends on the spatial position of each core inside the MCF. The maximum curvature sensitivity is reached when a pair of cores are aligned to the curvature direction. For the developed sensor the maximum sensitivity is $4.85 \mathrm{~nm} / \mathrm{m}^{-1}$.

\section{REFERENCES}

[1] J. Kong, X. Ouyang, A. Zhou, H. Yu, and L. Yuan, "Pure Directional Bending Measurement with a Fiber Bragg Grating at the Connection Joint of Eccentric-Core and Single-Mode Fibers," Journal of Lightwave Technology, vol. 34, no. 14, pp. 3288-3292, 2016.

[2] Q. Zhou, W. Zhang, L. Chen, Z. Bai, L. Zhang, L. Wang, B. Wang, and T. Yan, "Bending Vector Sensor Based on a Sector-Shaped LongPeriod Grating," IEEE Photonics Technology Letters, vol. 27, no. 7, pp. 713-716, Jan. 2015.

[3] T. Allsop, A. Gillooly, V. Mezentsev, T. Earthgrowl-Gould, R. Neal, D. Webb, and I. Bennion, "Bending and Orientational Characteristics of Long Period Gratings Written in D-Shaped Optical Fiber," IEEE Transactions on Instrumentation and Measurement, vol. 53, no. 1, pp. 130-135, 2004.

[4] T. Allsop, M. Dubov, A. Martinez, F. Floreani, I. Khrushchev, D. J. Webb, and I. Bennion, "A long period grating directional bend sensor incorporating index modification of the cladding," 17th International Conference on Optical Fibre Sensors, 2005.

[5] P. Saffari, T. Allsop, A. Adebayo, D. Webb, R. Haynes, and M. M. Roth, "Long period grating in multicore optical fiber: an ultra-sensitive vector bending sensor for low curvatures," Optics Letters, vol. 39, no. 12, p. 3508, Jun. 2014.

[6] H. Zhang, Z. Wu, P. P. Shum, R. Wang, X. Q. Dinh, S. Fu, W. Tong, and M. Tang, "Fiber Bragg gratings in heterogeneous multicore fiber for directional bending sensing," Journal of Optics, vol. 18, no. 8, p. 085705, 2016.

[7] Y. Awaji, J. Sakaguchi, B. J. Puttnam, R. S. Luís, J. M. D. Mendinueta, W. Klaus, and N. Wada, "High-capacity transmission over multi-core fibers," Optical Fiber Technology, vol. 35, pp. 100-107, 2017.

[8] I. Gasulla, D. Barrera, J. Hervás, and S. Sales, "Spatial Division Multiplexed Microwave Signal processing by selective grating inscription in homogeneous multicore fibers," Scientific Reports, vol. 7, p. $41727,2017$.

[9] L. Yuan and X. Wang, "Four-beam single fiber optic interferometer and its sensing characteristics," Sensors and Actuators A: Physical, vol. 138, no. 1, pp. 9-15, 2007.

[10] J. E. Antonio-Lopez, G. Salceda-Delgado, A. V. Newkirk, A. Schulzgen, and R. Amezcua-Correa, "Multiplexed High Temperature Sensor Based on Multicore Fiber," Advanced Photonics, 2014.

[11] L. Gan, R. Wang, D. Liu, L. Duan, S. Liu, S. Fu, B. Li, Z. Feng, H. Wei, W. Tong, P. Shum, and M. Tang, "Spatial-Division Multiplexed Mach-Zehnder Interferometers in Heterogeneous Multicore Fiber for Multiparameter Measurement," IEEE Photonics Journal, vol. 8, no. 1, pp. 1-8, 2016.

[12] J. P. Moore and M. D. Rogge, "Shape sensing using multi-core fiber optic cable and parametric curve solutions," Optics Express, vol. 20, no. 3, p. 2967, 2012.

[13] M. Gander, W. Macpherson, R. Mcbride, J. Jones, L. Zhang, I. Bennion, P. Blanchard, J. Burnett, and A. Greenaway, "Bend measurement using Bragg gratings in multicore fibre," Electronics Letters, vol. 36, no. 2, p. 120, 2000.

[14] A. Fender, W. N. Macpherson, R. R. J. Maier, J. S. Barton, D. S. George, R. I. Howden, G. W. Smith, B. J. S. Jones, S. Mcculloch, X. Chen, R. Suo, L. Zhang, and I. Bennion, "Two-Axis TemperatureInsensitive Accelerometer Based on Multicore Fiber Bragg Gratings," IEEE Sensors Journal, vol. 8, no. 7, pp. 1292-1298, 2008.

[15] R. M. Silva, M. S. Ferreira, J. Kobelke, K. Schuster, and O. Frazão, "Simultaneous measurement of curvature and strain using a suspended multicore fiber," Optics Letters, vol. 36, no. 19, p. 3939, 2011.

[16] D. Barrera, I. Gasulla, and S. Sales, "Multipoint Two-Dimensional Curvature Optical Fiber Sensor Based on a Nontwisted Homogeneous Four-Core Fiber," Journal of Lightwave Technology, vol. 33, no. 12, pp. 2445-2450, 2015.

[17] J. Madrigal, D. Barrera, J. Hervás, H. Chen, and S. Sales, "Directional curvature sensor based on long period gratings in multicore optical fiber," in Proc. SPIE 10323, 25th International Conference on Optical Fiber Sensors, Jeju, Korea, 2017, 103233A.

[18] M. Gallagher and U. Österberg, "Time resolved $3.10 \mathrm{eV}$ luminescence in germanium-doped silica glass," Applied Physics Letters, vol. 63, no. 22, pp. 2987-2989, 1993.

[19] T. Komukai and M. Nakazawa, "Fabrication of high-quality long-fiber Bragg grating by monitoring $3.1-\mathrm{eV}$ radiation $(400 \mathrm{~nm})$ from $\mathrm{GeO}$ defects," IEEE Photonics Technology Letters, vol. 8, no. 11, pp. 1495$1497,1996$.

[20] V. Bhatia, "Applications of long-period gratings to single and multiparameter sensing," Optics Express, vol. 4, no. 11, p. 457, 1999.

[21] X. Shu, L. Zhang, and I. Bennion, "Sensitivity characteristics of longperiod fiber gratings," Journal of Lightwave Technology, vol. 20, no. 2, pp. 255-266, 2002.

[22] U. Block, V. Dangui, M. Digonnet, and M. Fejer, "Origin of apparent resonance mode splitting in bent long-period fiber gratings," Journal of Lightwave Technology, vol. 24, no. 2, pp. 1027-1034, 2006. 